\title{
NeuroImage
}

\section{Functional connectivity in an fMRI working memory task in high-functioning autism}

\author{
Hideya Koshino, ${ }^{\mathrm{a}, \mathrm{b}, *}$ Patricia A. Carpenter, ${ }^{\mathrm{c}}$ Nancy J. Minshew, ${ }^{\mathrm{d}}$ Vladimir L. Cherkassky, \\ Timothy A. Keller, ${ }^{\mathrm{a}}$ and Marcel Adam Just ${ }^{\mathrm{a}}$ \\ ${ }^{a}$ Center for Cognitive Brain Imaging, Carnegie Mellon University, Pittsburgh, PA 15213, USA \\ ${ }^{\mathrm{b}}$ Department of Psychology, California State University, San Bernardino, CA 92407, USA \\ ${ }^{\mathrm{c}}$ Department of Psychology, Carnegie Mellon University, Pittsburgh, PA 15213, USA \\ ${ }^{\mathrm{d}}$ Department of Psychiatry and Neurology, School of Medicine, University of Pittsburgh, Pittsburgh, PA 15213, USA
}

Received 28 May 2004; revised 3 September 2004; accepted 21 September 2004

Available online 24 November 2004

\begin{abstract}
An fMRI study was used to measure the brain activation of a group of adults with high-functioning autism compared to a Full Scale and Verbal IQ and age-matched control group during an n-back working memory task with letters. The behavioral results showed comparable performance, but the fMRI results suggested that the normal controls might use verbal codes to perform the task, while the adults with autism might use visual codes. The control group demonstrated more activation in the left than the right parietal regions, whereas the autism group showed more right lateralized activation in the prefrontal and parietal regions. The autism group also had more activation than the control group in the posterior regions including inferior temporal and occipital regions. The analysis of functional connectivity yielded similar patterns for the two groups with different hemispheric correlations. The temporal profile of the activity in the prefrontal regions was more correlated with the left parietal regions for the control group, whereas it was more correlated with the right parietal regions for the autism group.

(C) 2004 Elsevier Inc. All rights reserved.
\end{abstract}

Keywords: Autism; fMRI; Working memory

\section{Introduction}

In the present study, we were interested in the cortical networks used by individuals with autism during an n-back task with letter stimuli. Previous studies in autism have converged on a number of points; one of which is that individuals with autism tend to rely on lower level rather than higher level processing (e.g., Mottron et al.,

* Corresponding author. Department of Psychology, California State University, 5500 University Parkway, San Bernardino, CA 92407, USA. Fax: +19098807003 .

E-mail address: hkoshino@csusb.edu (H. Koshino).

Available online on ScienceDirect (www.sciencedirect.com).
2001). This tendency can result in good performance on tasks requiring analysis of visuospatial details, such as the Block Design and Object Assembly subtests of the Wechsler Intelligence Scale (WAIS) (e.g., Frith, 1989; Shah and Frith, 1983), perceptual learning tasks (Plaisted et al., 1998a), visual search tasks (O'Riordan et al., 2001; Plaisted et al., 1998b), and global precedence tasks (Plaisted et al., 1999). On the other hand, this tendency may be less adaptive in other types of tasks that require processing of more complex information such as language comprehension (e.g., Minshew et al., 1997). In this regard, brain imaging studies have shown that participants with autism seem to show less activation in the regions related to higher level cognition and more activation in the regions associated with lower level cognition compared to control participants (e.g., Just et al., 2004; Ring et al., 1999). This pattern is consistent with the conclusion that people with autism prefer visually based processing styles, which are associated with more activation in the posterior brain regions, while they may not be good in using higher level working memory and language, which are associated more with the anterior cortical regions. A second point that emerges from the literature is that individuals with autism seem to show more right hemisphere activation than left (e.g., Boddaert and Zilbovicius, 2002; Muller et al., 1999). This tendency might be a further reflection of their preference for lower level nonverbal feature analyses over higher level language-based processing styles. We examined these issues in an n-back letter working memory task in which Working Memory Load was manipulated, while the visual information of the letter sequences remained constant across different n-back conditions.

We were also interested in examining the temporal dimension of processing for individuals with autism and their controls by using a functional connectivity measure, the correlation between the temporal profiles of activity between cortical regions (e.g., Friston et al., 1993; McIntosh and Gonzalez-Lima, 1994). An fMRI study of sentence comprehension (Just et al., 2004), for example, suggested that individuals with autism may have 
quantitatively lower functional connectivity than their control counterparts, especially in the regions related to the integration of information. What makes it interesting to explore this issue in the context of the n-back task is that the task is performed well by both high-functioning individuals with autism and controls (Williams et al., in press $(\mathrm{a}, \mathrm{b}))$, so comparisons are less confounded by behavioral differences. If these measures help identify functional systems, then the large-scale cortical networks (e.g., Mesulam, 1998) of people with autism may be (1) more active in posterior regions related to lower level cognition, (2) more right lateralized, and (3) have lower functional connectivity.

Autism is identified with symptoms including impairments in social interaction, impairments in verbal and nonverbal communication, and stereotypic or repetitive behaviors (American Psychiatric Association, 1994). However, some individuals with autism may show better performance in tasks such as visuospatial information processing, rote memory, and calculation. Also, people with autism may show better performance relative to controls with simpler tasks across domains, such as simple attention, memory, language, or visuospatial tasks, than with more complex tasks such as skilled motor, complex memory, complex language, and reasoning domains (Minshew et al., 1997).

Some studies have reported that individuals with autism show differences between verbal and visuospatial information processing in intelligence scales, such as Wechsler Adult Intelligence Scale (WAIS) and Wechsler Intelligence Scale for Children (WISC), with higher scores for nonverbal performance tasks such as Block Design and Object Assembly than for verbal tasks (e.g., Frith, 1989; Shah and Frith, 1983, 1993), although other researchers have not found such a tendency in high-functioning autism (Siegel et al., 1996). Furthermore, some studies have found that people with autism showed better performance than, or at least different processing from, normal controls in other visuospatial tasks, such as perceptual learning (Plaisted et al., 1998a), visual search (O'Riordan et al., 2001; Plaisted et al., 1998b), and global precedence (Plaisted et al., 1999). These researchers suggested that people with autism tend to process low-level visual features (details) but may not be able to integrate features into global structures reflecting the hierarchical nature of the environmental stimuli; therefore, it is difficult for them to find the central meaning of the environmental stimuli (e.g., Hill and Frith, 2003).

In an fMRI study of the Embedded Figures Test (EFT), participants were asked to find a target figure embedded in a complex visual pattern (Ring et al., 1999). The control group showed activation in the prefrontal regions that were not activated in the autism group, whereas the autism group showed more activation in ventral occipitotemporal regions. The authors concluded that individuals with autism rely on visuospatial feature analysis rather than working memory, while normal controls utilize working memory in EFT. However, individuals with autism do not necessarily show better performance with all kinds of visual stimuli. For example, they usually do not perform well in face recognition tasks and tend to show less activation in the fusiform face areas (e.g., Critchley et al., 2000; Pierce et al., 2001; Schultz et al., 2000).

Neuroimaging studies of language processing also suggest differences between the brain activity of individuals with autism and matched controls. For example, a PET study compared autism and control groups in tasks including listening to sentences, repeating sentences, and generating sentences (Muller et al., 1999). For the autism group, the left dorsolateral prefrontal cortex, thalamus, and right dentate nucleus showed less activation for receptive and expressive language conditions. On the other hand, the control group showed left hemisphere dominance, which is very typical in language-related tasks. In a sentence comprehension task using fMRI, control participants showed greater activation in Broca area, whereas the autism group showed greater activation in Wernicke area (Just et al., 2004). Also, the degree of temporal synchronization (functional connectivity) between activated voxels in these cortical areas was generally lower for the autism group. Regarding the hemispheric differences, some studies, using auditory verbal and nonverbal sounds, have found that people with autism tend to show more right hemisphere activation, whereas normal controls show more left hemisphere activation (e.g., Boddaert and Zilbovicius, 2002; Muller et al., 1999).

The neural correlates of working memory performance have received a great deal of attention using brain imaging techniques with unselected populations (e.g., Braver et al., 1997; Postle, 2000; Smith et al., 1998). Typically, the processing of verbal information activates more regions in the left hemisphere, whereas processing of nonverbal and spatial information seems to be associated with the right hemisphere (e.g., Owen et al., 1998; Smith and Jonides, 1999; Smith et al., 1998), although these divisions are not absolute and vary with the task and difficulty. Similarly, the organization of the prefrontal regions does not show a clear distinction corresponding to the dorsal-ventral distinction of the posterior visual regions (e.g., D'Esposito, 2001; Fletcher and Henson, 2001; Nystrom et al., 2000; Ungerleider and Haxby, 1984). Neither does there appear to be a clear division, within the prefrontal regions, for activation that corresponds to two proposed functions of working memory, namely, maintenance and manipulation. Brain imaging studies have found that maintenance tends to activate a more ventral part of the prefrontal cortex, whereas manipulation tends to activate a more dorsal part of prefrontal region; however, the division is not clear and varies with materials and difficulty (e.g., D'Esposito et al., 1999; Petrides, 1994).

With regard to individuals with autism and working memory tasks, the behavioral research has yielded mixed results that have led to a questioning of the concept of a unitary working memory. Some studies have found performance deficits in individuals with autism compared to normal controls. For example, Bennetto et al. (1996) compared autism and control groups in various memory tasks. The autism group showed a performance deficit in temporal order memory, source memory, sentence and digit span, and executive function tasks, such as the Wisconsin Card Sorting Task and the Tower of Hanoi, but not in short- and long-term recognition and cued recall. On the other hand, different results were reported by other researchers (Ozonoff and Strayer, 2001; Ozonoff et al., 1994; Russell et al., 1996) who tried to isolate specific executive functions using the Wisconsin Card Sorting Task (WCST), a go-no go task, and a global-local processing task. They found that individuals with autism performed as well as the control participants in tasks requiring inhibition of neutral stimuli and global-local processing; however, the autism group performed poorly in the tasks requiring cognitive flexibility and inhibition of prepotent responses. These researchers concluded that the mixed findings about the working memory deficits may result from how working memory tasks are administered and that autism may not actually involve working memory deficits. In contrast, children and adults with autism do poorer than age- and cognitive-matched controls on spatial working memory tasks (Luna et al., 2002; Williams et al., in press(a,b)). Luna et al. (2002) used fMRI to 
examine spatial working memory with an oculomotor delayedresponse task and a visually guided saccade task. The autism and control groups performed similarly in the visually guided saccade task, but the autism group showed less activation in the dorsolateral prefrontal cortex and the posterior cingulate in the oculomotor delayed-response task. They concluded that people with autism have an impairment in spatial working memory.

Brain imaging techniques also enable the investigation of the functional connectivity or the synchronization of cortical activation in autism. For example, using a theory of mind task, Castelli et al. (2002) found weaker connectivity between V3 and the superior temporal sulcus in autism. They suggested that this weaker connectivity may reflect a lack of top-down modulation from more anterior regions such as the amygdala and surrounding temporal pole and/or medial prefrontal cortex. Just et al. (2004) also showed that functional connectivity was lower for the autism than for the control group in a sentence comprehension task. They proposed the underconnectivity theory of autism which claims that autism is a cognitive and neurobiological disorder marked and caused by underdevelopment of the integrative circuitry that results in a deficit of integration of information at the neural and cognitive levels. According to the theory, the cognitive deficit in autism is most likely to arise when the task requires integrative processing (i.e., an emergent process) at a higher cognitive level. The theory predicts that any facet of psychological or neurological function that is dependent on the coordination or integration of brain regions is susceptible to disruption, particularly when the computational demand of the coordination is high.

The main goals of this study are to investigate three characteristics of information processing of individuals with autism in an nback letter working memory task. It was hypothesized that the autism group would show (1) more activation in the posterior regions related to lower level feature analysis and less activation in the anterior regions that are related to higher level cognition requiring integration of information. It was also predicted that the autism group would exhibit (2) greater processing of information in the right hemisphere as compared to the left hemisphere, indicating a greater use of nonverbal strategies of information processing as compared to verbal strategies. Finally, the autism group was expected to show (3) weaker functional connectivity (underconnectivity) in the large-scale brain network.

\section{Methods}

\section{Participants}

The participants were 14 high-functioning individuals with autism (13 males and 1 female) and 14 healthy normal control participants (13 males and 1 female). They were matched for age (autism group mean: 25.7 and control group mean: 29.8, $t(26)=$ $1.03, \mathrm{~ns})$, Full Scale and Verbal IQ as determined by the Wechsler Adult Intelligence Scale Revised (WAIS-R, 80 or above), and gender. The average Full Scale IQ was 100.1 for the autism group and 109.1 for the control group, $t(26)=2.00, \mathrm{~ns}$, and the average Verbal IQ was 102.6 for the autism group and 108.3 for the control group, $t(26)=1.16$, ns.

The diagnosis of autism was established using two structured research diagnostic instruments supplemented with expert clinical application of accepted criteria of high-functioning autism
(Minshew, 1996). All participants in the autism group met the cutoffs for autism as determined by the developers of the ADOS (Autism Diagnostic Observation Schedule, Lord et al., 1989, 2000) for the Communication (cutoff 3; range 3-6), Reciprocal Social Interaction (cutoff 6; range 6-13), and Total algorithm scores (cutoff 10; range 10-19). Participants who received scores in the autism spectrum range outside of the strict autism cutoff on this diagnostic measure were excluded from this study. In addition, all participants with autism met cutoffs for autism on the ADI (Autism Diagnostic Interview, Le Courteur et al., 1989; Lord et al., 1994), which assesses developmental history and reported current functioning, for Reciprocal Social Interaction and Communication and had abnormal development before 3 years of age. They also all met the cutoff for Restricted, Repetitive, and Stereotyped Behaviors except for one participant who was within one point of the cutoff for this area.

Potential participants with autism were excluded on the basis of an associated infectious, genetic, or metabolic disorder, such as fragile-X syndrome, tuberous sclerosis, or fetal cytomegalovirus infection. Potential control and autism participants were also excluded in cases of evidence of birth asphyxia, head injury, or a seizure disorder. Other exclusionary criteria were based on neurologic history and examination, any type of antiseizure medication, and chromosomal analysis or metabolic testing if indicated. Participants with autism were recruited from autism conferences, Web sites, and parent support groups, and tended to be volunteers primarily from middle or higher socioeconomic levels.

The control participants were community volunteers recruited to match the autism participants on age, Full Scale IQ, gender, race, and socioeconomic status of family of origin, as measured by the Hollingshead method (Hollingshead, 1957). Potential control participants were screened by questionnaire, telephone, face-toface interview, and observation during screening psychometric tests. Exclusionary criteria, evaluated through these procedures, included current or past psychiatric and neurologic disorders, birth injury, developmental delay, school problems, acquired brain injury, learning disabilities, substance abuse, and medical disorders with implications for the central nervous system (such as extreme use of steroidals in inhalers for asthmatics) or those requiring regular medication (such as high blood pressure or diabetes). Potential control participants were also screened to exclude those with a family history (in parents, siblings, and offspring) of autism, developmental cognitive disorders, affective disorders, anxiety disorders, schizophrenia, obsessive compulsive disorder, substance abuse, or other neurologic or psychiatric disorder thought to have a genetic component.

Handedness was determined with the Lateral Dominance Examination from the Halstead-Reitan Neuropsychological Test Battery (Reitan, 1985), revealing that three participants with autism and one control participant were left-handed. The brain activation data from these left-handers were clearly similar to their respective groups, and therefore, the data are not separated by handedness. Five of the autism participants were taking medication (three of these were taking selective serotonin uptake inhibitors, one of these was also taking antianxiety medications, one was taking allergy medications, and one was taking high blood pressure medications). The data of all these individuals were qualitatively similar to the presented data of the participants with autism without medication. Three of the control participants were taking allergy, gastrointestinal, anti-infection, and hair loss prevention medications. 
Their data were also similar to those of the other controls. Written informed consent was obtained from participants or their guardians, using procedures approved by the University of Pittsburgh Medical Center and Carnegie Mellon University Institutional Review Boards.

\section{Experimental paradigm}

Participants performed a letter n-back task with three experimental conditions: 0-back, 1-back, and 2-back. In the 0-back condition, participants were asked to remember a target letter that was presented at the beginning of each trial block. A sequence of 20 letters was presented, and participants were instructed to press a response button when the target letter appeared on the screen. In the 1-back condition, participants were told to respond when the same letter was presented twice in a row, and in the 2-back condition, they responded when a letter matched one that had been presented two letters ago. Stimulus letters in each trial were randomly selected from among 15 letters: A, F, H, I, J, K, L, M, O, $\mathrm{P}, \mathrm{Q}, \mathrm{R}, \mathrm{S}, \mathrm{U}$, and Y.

Stimuli were projected onto a viewing screen attached within the bore of the scanner and viewed at a distance of approximately $20 \mathrm{~cm}$ from the participant's eyes through two mirrors positioned on top of the head coil. A fiber optic button box was used for participants to signal their responses. Stimulus presentation and behavioral data collection were controlled with custom-written experimental presentation software on a PC running Windows NT.

The experiment utilized a blocked design with four epochs for each of the three experimental conditions (12 epochs total) with 20 letters per epoch and targets occurring four times in each epoch. At the beginning of each epoch, a visual instruction indicated the condition (0-back, 1-back, or 2-back) for $5 \mathrm{~s}$. Each stimulus letter was presented for $500 \mathrm{~ms}$, followed by 1-s delay (see Fig. 1). The epochs were ordered in an ABCCBA fashion. A fixation epoch, in which three asterisks for fixation $(* * *)$ were presented for $24 \mathrm{~s}$, was inserted after every three epochs and at the beginning and end of the session, resulting in a total of five fixation epochs. The data from these fixation epochs were used as the baseline condition. The entire functional scanning run took about $12 \mathrm{~min}$.

\section{Imaging parameters}

Scanning was done in a 3.0-T GE Medical Systems scanner (Thulborn et al., 1996) at the University of Pittsburgh Magnetic Resonance Research Center. A T2*-weighted single-shot spiral pulse-sequence sensitive to blood oxygen level-dependent (BOLD, see Kwong et al., 1992; Ogawa et al., 1990) contrast was used with the following acquisition parameters: $\mathrm{TR}=1000 \mathrm{~ms}$, TE $=18 \mathrm{~ms}$,

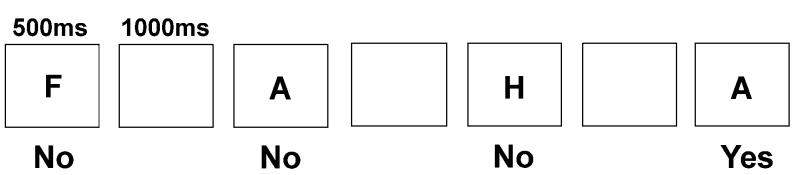

Fig. 1. Schematic representation of the 2-back condition. Each stimulus letter is presented for $500 \mathrm{~ms}$ then followed by a blank display for $1000 \mathrm{~ms}$. Participants were asked to judge if each letter is the same as the one that appeared two letters ago.

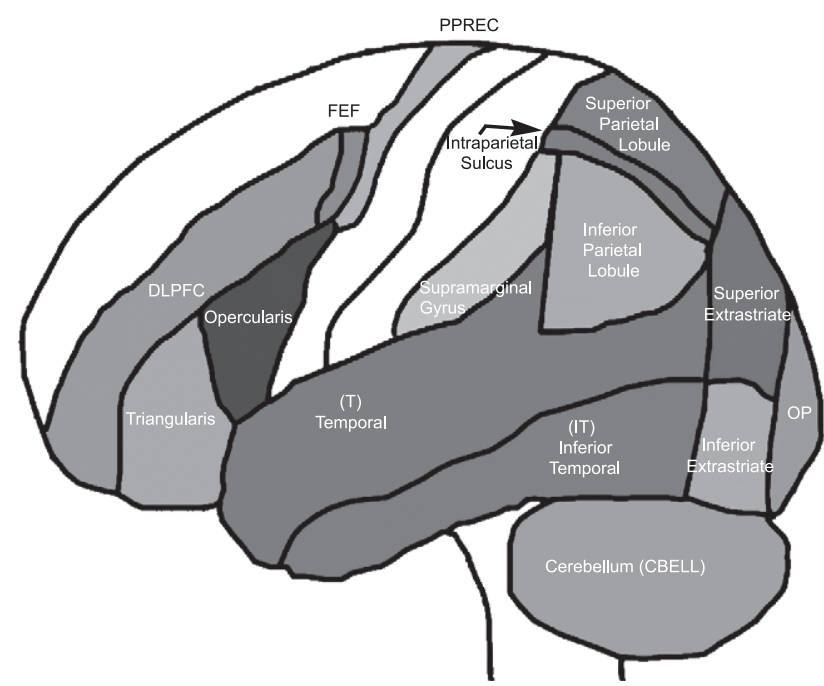

Fig. 2. Schematic diagram of cortical parcellation scheme based on Rademacher et al. (1992). Not shown in this view are four more medial ROIs, namely, superior medial frontal paracingulate, anterior cingulate, calcarine sulcus, and supplementary motor area.

flip angle $=70^{\circ}, \mathrm{FOV}=20 \times 20 \mathrm{~cm}$, matrix size $=64 \times 64$, axialoblique plane with 16 slices, and a voxel size of $3.125 \times 3.125 \times 5$ $\mathrm{mm}$ with a 1-mm gap. High-resolution T1-weighted structural images were acquired with a 3D SPGR volume scan with the following parameters: $\mathrm{TR}=25, \mathrm{TE}=4$, flip angle $=40^{\circ}$, $\mathrm{FOV}=$ $24 \times 18 \mathrm{~cm}, 124$ slices, resulting in voxel dimensions of $0.9375 \times$ $0.9375 \times 1.5 \mathrm{~mm}$ thick, taken axially.

\section{fMRI data analysis}

Image preprocessing, including baseline correction, mean correction, motion correction, and trend correction, was performed using FIASCO (Eddy et al., 1996; Lazar et al., 2001; further description and tools are available at www.stat.cmu.edu/ fiasco/. The maximum in-plane displacement estimate between individual images and a reference image did not exceed 0.3 voxels.

To compare the amount of activation across the experimental conditions in various regions, anatomical ROIs were defined individually for each participant by adapting the parcellation scheme of Rademacher et al. (1992) (Caviness et al., 1996), as shown in Fig. 2. This method uses limiting sulci and coronal planes, defined by anatomical landmarks, to segment cortical regions. Because each individual's cortical anatomy is different, the ROIs were drawn on the structural images of each participant. Coregistration was done by first computing the mean functional image for each of the functional slices. These mean images were then registered in parallel alignment with the anterior commissureposterior commissure (AC-PC) line to a structural volume scan of each participant. The limiting sulci and other anatomical landmarks were then located by viewing the structural images simultaneously in the three orthogonal planes. The ROIs were defined by manually tracing the regions onto the axial image of each functional slice. The interrater reliability of this ROI-defining procedure between two trained staff members was previously evaluated for four ROIs in two participants in another study (Just et al., 2001). The reliability measure was obtained by dividing the size of the set of 
voxels that overlapped between the two raters by the mean of their two set sizes. The resulting eight reliability measures were in the $78-91 \%$ range, with a mean of $84 \%$, as high as the reliability reported by the developers of the parcellation scheme. This method allows us to measure the modulation of the activation by the independent variables in regions that are specified a priori and require no morphing for definition. In addition, anatomical ROIs localize the activation in individual participant's brains more accurately than whole-brain normalization into a common brain space (Nieto-Castanon et al., 2003). The main drawback of reliably defining anatomical ROIs for each participant is the expense of having two highly trained staff members defining and checking many ROIs in each brain.

Data from the 6-s rest interval and from the first $6 \mathrm{~s}$ of each block of trials were discarded to accommodate the rise and fall of the hemodynamic response (Bandettini et al., 1992). To identify active voxels, voxelwise $t$ tests were performed to compare a voxel's mean signal intensity in each experimental condition with that of the fixation condition. A $t$ threshold was set individually for each participant such that each one had exactly a total of 160 activated voxels summed across all the ROIs, excluding the cerebellum and occipital pole, for the 2-back condition. The goal of this normalization procedure was to equate the level of activation between the two groups, and it allowed us to compare how the activation was distributed for the two groups across the ROIs. The cerebellum and occipital pole were excluded as areas with potentially high level of susceptibility artifacts. The total number of voxels (160) was selected because that was the average number of voxels in these areas for both groups when the same data were analyzed with a fixed $t$ threshold of 5.5 for all subjects. The selected level of activation (160 voxels) was appropriate for all participants as it did not admit obvious noise voxels in any of the experimental conditions, and it resulted in activation volumes and threshold values that are consistent with other fMRI studies of similar tasks. The average $t$ threshold was 5.49 for the autism group and 5.28 for the control group, $t(26)=0.53$, ns. The general results were similar when all participants were analyzed with a $t$ threshold of 5.5.

The main dependent measure was the sum of the percentage change in signal intensity (ssi) of the voxels activated above each participant's individually chosen threshold. This measure takes into account both the spatial extent of activation and the amplitude, and it was computed for each ROI and each participant in each condition. These data were then submitted to a 2 (Group) $\times 3$ (Working Memory Load) mixed analysis of variance (ANOVA) for each ROI. Also, the Talairach coordinate (Talairach and Tournoux, 1988) was computed for the mean centroid of activation for each ROI for the 2-back condition.

\section{Functional connectivity}

Functional connectivity refers to a correlation or synchronization between the time courses of activation of two regions. The idea behind functional connectivity analysis is that regions that work together have similar temporal response profiles; therefore, a correlation coefficient between the activations of these regions across the time course should be high (e.g., Friston et al., 1993; McIntosh and Gonzalez-Lima, 1994). To compute the measure of functional connectivity, the processed data were first Fourierinterpolated in time to correct for the interleaved slice acquisition sequence. For each participant, a mean time-course was computed across activated voxels in each ROI if there were three or more activated voxels in the ROI. A correlation coefficient was then calculated between the time courses of pairs of ROIs. There were 40-43 images in each epoch, depending on condition, and 4 epochs per condition; however, the correlations were based on 119-127 observations, because some images were excluded from the analysis due to excessive head motion. These correlation coefficients were then transformed using Fisher $r$-to- $z^{\prime}$ transformation, and the mean $z^{\prime}$-transformed values were computed across participants for each group and for each ROI pair. The mean $z^{\prime}$-transformed values were then converted back to correlation coefficients, and a correlation matrix was created for each group. ROIs were excluded from the correlation matrix if less than two participants had three or more active voxels in the ROI. An exploratory factor analysis (e.g., McLaughlin et al., 1992; Peterson et al., 1999) was then performed for each group separately. Only the 2-back condition was used for the factor analysis because the activation was low for the 0 - and 1-back conditions, and therefore there were not enough ROI pairs in the correlation matrices. Our logic behind the factor analyses was that each factor would represent a large-scale network among brain regions corresponding to some functions (e.g., Mesulam, 1990, 1998). Factors that had eigenvalues of 1.0 or above were retained. In this case, an eigenvalue corresponds to the equivalent number of ROIs that the factor represents. Factor loadings represent the degree to which each of the ROIs correlates with each of the factors, and ROIs that had factor loadings of 0.4 or greater were taken into consideration in interpretation.

\section{Results}

\section{Behavioral data}

The mean response time (RT) and error rate data are shown in Table 1. The behavioral data from one participant in the control group were lost due to computer malfunction; therefore, the control group consisted of 13 participants and the autism group of 14 participants. The data were submitted to a 2 (Group) $\times 3$ (Working Memory Load) mixed analysis of variance (ANOVA). As is seen in Table 1, the performance between the control and autism groups was very similar to each other and resulted in no significant group difference in both the RT and error data, $F(1,25)=0.82$, ns, and $F(1,25)=0.64$, ns, respectively. Both groups showed increases with increased memory load for RT, $F(2,50)=9.08, P<0.001$, and for the error rate $F(2,50)=15.47, P<0.001$. Neither RT nor error rate showed significant interaction between the Group and Work-

Table 1

Mean response time (ms) and error rate (\%)

\begin{tabular}{lllr}
\hline & 0-Back & 1-Back & \multicolumn{1}{c}{ 2-Back } \\
\hline $\begin{array}{l}\text { Response time (ms) } \\
\text { Autism, mean (SE) }\end{array}$ & $462(16.9)$ & $474(16.8)$ & $541(35.8)$ \\
Control, mean (SE) & $490(21.3)$ & $515(25.3)$ & $547(24.1)$ \\
& & & \\
Error Rate (\%) & & & \\
Autism, mean (SE) & $0.4(0.5)$ & $2.8(1.2)$ & $9.8(2.5)$ \\
Control, mean (SE) & $4.3(2.6)$ & $2.1(1.2)$ & $11.1(2.6)$ \\
\hline
\end{tabular}


ing Memory Load, $F(2,50)=0.60$, ns, and $F(2,50)=0.93$, ns, respectively.

There was no evidence of the autism group having difficulty in task switching between the various experimental conditions. In fact, the mean RT on only the first positive item in a new type of block (e.g., 1-back following 2-back) was slightly shorter for the autism group than for the control group.

\section{Amount of brain activation}

The ANOVA results for the sum of the percentage change in signal intensity (ssi) in the ROIs and the mean centroids are shown in Table 2. There are three main findings in the sum of signal intensity data. First, the autism group showed less activation in some left hemisphere prefrontal and parietal regions than the controls for the 2-back condition, resulting in significant Group $\times$ Memory Load interactions. These regions included the left dorsolateral prefrontal cortex, $F(2,52)=4.33, P<0.05$, the left inferior frontal gyrus, $F(2,52)=7.03, P<0.01$, the left posterior precentral sulcus, $F(2,52)=4.99, P<0.05$, and the left inferior parietal lobe, $F(2,52)=4.84, P<0.05$. Second, the autism group showed higher activation than the control group in some right hemisphere ROIs, including the right inferior frontal gyrus, $F(1,26)=5.96, P<0.05$ and the right inferior parietal lobe, $F(1,26)=5.59, P<0.05$. Third, the autism group also showed more activation than the control group in the posterior ROIs such as the left inferior temporal, $F(1,26)=3.71, P<0.07$, the left temporal lobe, $F(1,26)=4.32, P<0.05$, the right temporal,
$F(1,26)=5.18, P<0.05$, and the left inferior extrastriate, $F(1,26)=$ $5.19, P<0.05$. In these posterior ROIs, the control group showed very little activation. These results are shown in Table 2 and Fig. 3. Fig. 4 shows the $t$ maps of the representative ROIs. Overall, both groups showed a similar level of brain activation due to the normalization as discussed in Methods.

\section{Functional connectivity}

The results of the factor analyses, seen in Table 3 and Fig. 5, showed that the autism group has three factors. Factor 1 consists of the prefrontal and right parietal ROIs, including the left and right dorsolateral prefrontal cortex and the right inferior parietal lobe. This factor was interpreted as representing a network related to working memory. Factor 2 contains primarily the left parietal ROIs and the premotor regions. Factor 3 includes the posterior ROIs, such as the inferior temporal and occipital lobes, corresponding to a network for the visual feature analysis. The control group has two factors. Factor 1 consists of the frontal and left parietal ROIs, representing a working memory network. Factor 2 centers around the right hemisphere parietal ROIs. These factors accounted for approximately $60 \%$ of the total variance for both groups.

There are three main differences between the network structures of the two groups. One is the difference between the two working memory networks. In the autism group, the left and right frontal regions are closely synchronized with the right parietal regions, whereas in the control group, the left and right frontal regions are more related to the left parietal regions. The second difference is

Table 2

Sum of percentage change in signal intensity and mean $x y z$ coordinates

\begin{tabular}{|c|c|c|c|c|c|c|c|c|c|c|c|c|}
\hline & \multicolumn{6}{|c|}{ Sum of percentage change in signal intensity } & \multicolumn{6}{|c|}{ Centroids } \\
\hline & \multicolumn{3}{|l|}{ Autism } & \multicolumn{3}{|l|}{ Control } & \multicolumn{3}{|c|}{ Autism } & \multicolumn{3}{|c|}{ Control } \\
\hline & 0 -Back & 1-Back & 2-Back & 0 -Back & 1-Back & 2-Back & $x$ & $\mathrm{y}$ & $\mathrm{z}$ & $\mathrm{x}$ & $\mathrm{y}$ & $\mathrm{z}$ \\
\hline L dorsolateral prefrontal cortex ${ }^{i}$ & 2.8 & 8.7 & 16.4 & 4.8 & 10.4 & 32.4 & 32 & -28 & 35 & 32 & -35 & 32 \\
\hline $\mathrm{R}$ dorsolateral prefrontal cortex & 3.5 & 12.3 & 30.8 & 6.2 & 7.8 & 35.6 & -33 & -27 & 39 & -35 & -27 & 36 \\
\hline L frontal eye field & 0.5 & 1.8 & 5.3 & 1.3 & 2.2 & 10.2 & 41 & -3 & 48 & 41 & -3 & 42 \\
\hline $\mathrm{R}$ frontal eye field & 1.5 & 5.4 & 11.9 & 1.3 & 2.7 & 8.8 & -42 & -6 & 46 & -44 & -5 & 48 \\
\hline $\mathrm{L}$ inferior frontal gyrus ${ }^{\mathrm{i}}$ & 1.6 & 4.6 & 5.8 & 1.1 & 2.0 & 12.9 & 37 & -17 & 21 & 41 & -16 & 23 \\
\hline $\mathbf{R}$ inferior frontal gyrus ${ }^{\mathrm{g}}$ & 1.4 & 8.3 & 14.6 & 0.9 & 0.7 & 8.1 & -46 & -16 & 20 & -45 & -14 & 21 \\
\hline $\mathrm{L}_{\text {posterior precentral sulcus }}{ }^{\mathrm{i}}$ & 2.7 & 4.2 & 8.2 & 2.6 & 4.0 & 15.2 & 40 & 4 & 52 & 39 & 5 & 51 \\
\hline $\mathrm{R}$ posterior precentral sulcus & 3.2 & 7.2 & 13.7 & 2.9 & 5.2 & 12.9 & -43 & 4 & 49 & -40 & 3 & 50 \\
\hline Supplementary motor area & 5.8 & 5.2 & 10.4 & 2.5 & 2.8 & 9.4 & -2 & 6 & 64 & 0 & 5 & 64 \\
\hline Superior medial frontal paracingulate & 5.2 & 6.1 & 18.5 & 4.3 & 5.9 & 27.1 & -4 & -12 & 55 & -1 & -12 & 53 \\
\hline $\mathbf{L}$ inferior parietal lobe $\mathrm{e}^{\mathrm{i}}$ & 2.8 & 6.2 & 12.3 & 3.7 & 6.5 & 21.3 & 47 & 46 & 39 & 46 & 50 & 42 \\
\hline$R$ inferior parietal lobe $e^{g}$ & 5.2 & 10.0 & 21.9 & 2.0 & 2.2 & 10.4 & -47 & 47 & 38 & -44 & 49 & 39 \\
\hline $\mathrm{L}$ intraparietal sulcus & 3.9 & 9.8 & 28.5 & 4.2 & 8.5 & 34.4 & 34 & 52 & 46 & 32 & 57 & 46 \\
\hline $\mathrm{R}$ intraparietal sulcus & 6.8 & 15.5 & 41.9 & 6.0 & 7.9 & 28.6 & -32 & 56 & 49 & -34 & 56 & 48 \\
\hline L superior parietal lobe & 0.5 & 1.2 & 8.6 & 0.4 & 1.3 & 5.7 & 17 & 62 & 49 & 18 & 60 & 55 \\
\hline $\mathrm{R}$ superior parietal lobe & 1.4 & 3.3 & 16.5 & 0.9 & 1.7 & 11.9 & -15 & 66 & 49 & -18 & 62 & 52 \\
\hline $\mathrm{L}$ inferior temporal & 2.0 & 5.6 & 8.0 & 0.5 & 1.6 & 4.9 & 47 & 54 & -2 & 48 & 60 & 2 \\
\hline $\mathrm{R}$ inferior temporal & 9.6 & 10.3 & 13.3 & 2.5 & 1.7 & 8.1 & -46 & 54 & -1 & -43 & 60 & -1 \\
\hline$L$ temporal $^{\mathrm{g}}$ & 2.0 & 4.3 & 6.5 & 0.4 & 0.6 & 1.1 & 56 & 18 & 7 & 52 & 28 & 12 \\
\hline $\mathrm{R}$ temporal $^{\mathrm{g}}$ & 2.6 & 3.7 & 6.1 & 0.5 & 0.5 & 1.2 & -53 & 30 & 9 & -46 & 25 & 9 \\
\hline$L$ inferior extrastriate $\mathrm{g}^{\mathrm{g}}$ & 1.9 & 2.5 & 4.8 & 0.0 & 0.2 & 2.0 & 34 & 63 & -5 & 37 & 67 & -5 \\
\hline $\mathrm{R}$ inferior extrastriate & 3.8 & 3.0 & 7.4 & 1.0 & 1.8 & 6.4 & -32 & 63 & -3 & -20 & 62 & -5 \\
\hline L superior extrastriate & 1.2 & 1.3 & 3.8 & 0.2 & 0.7 & 2.5 & 20 & 79 & 35 & 26 & 77 & 36 \\
\hline $\mathrm{R}$ superior extrastriate & 1.3 & 2.5 & 6.8 & 0.4 & 0.0 & 1.5 & -14 & 76 & 38 & -15 & 80 & 38 \\
\hline
\end{tabular}

Note. ROIs with bold-type font are the ones with statistically significant results at $P<0.05$ in the $2 \times 3$ mixed ANOVA. The nature of the effect is shown in the superscript on the right shoulder of the ROI names. g: group main effect and i: group $\times$ condition interaction. All ROIs in the table showed the significant or marginally significant condition main effect, except L temporal. 


\section{Dorsolateral PreFrontal Cortex}

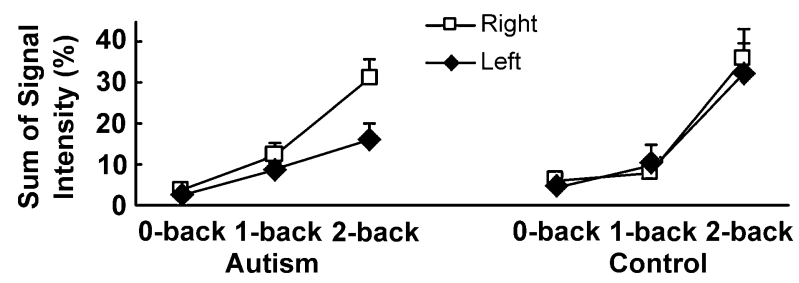

Inferior Parietal Lobe

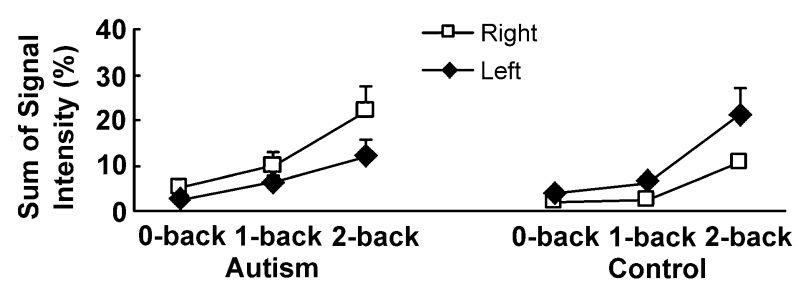

Inferior Temporal Gyrus

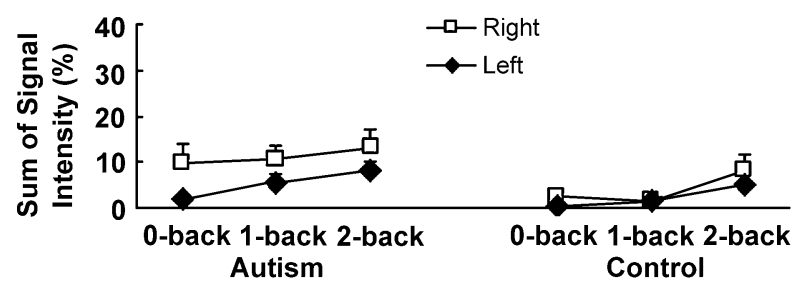

Fig. 3. Sum of signal percentage change in signal intensity plotted against the three working memory load conditions. The dorsolateral prefrontal cortex (DLPFC) and the inferior parietal (IPL) show significant Group $\times$ Memory Load interactions. For the left DLPFC, the interactions are caused by the lower activation of the autism group for the 2-back condition. For the IPL, the autism group showed more activation in the right hemisphere, whereas the control group showed more activation in the left hemisphere. For the inferior temporal, the autism group showed significantly more activation than for the control group. In the inferior temporal (IT), the autism group showed higher activation than the control group. The same pattern was observed in some other posterior ROIs, including the left and right temporal and the left inferior extrastriate.

that the working memory network seems to be smaller for the autism group involving fewer ROIs (8 ROIs) than for the control group (11 ROIs). The third difference is that, compared to the control group, the autism group showed less synchronized frontal activity, as indicated by the size of Factor 1, and more synchronized posterior activity, as indicated by the third factor representing the posterior visual network.

In addition to the factor analyses, functional connectivity was analyzed by directly comparing the Fisher $z^{\prime}$-transformed values between the two groups. The mean of the $z^{\prime}$-transformed values was computed for each ROI pair, then a $t$ test was performed to compare the autism with the control group. As shown in Fig. 6, significant group differences were found in the ROI pairs around the left inferior parietal lobe. The autism group showed lower correlations than the control group between the left inferior parietal and the right dorsolateral prefrontal cortex, $t(22)=2.01, P<0.06$; the right frontal eye fields, $t(10)=2.14, P<0.06$; the right posterior precentral sulcus, $t(14)=2.48, P<0.05$; the left intraparietal sulcus, $t(23)=2.05, P<0.055$; the right intraparietal sulcus, $t(23)=1.90, P<0.075$; and the right superior parietal, $t(17)=2.08, P<0.55$. The autism group also showed lower correlation between the left intraparietal sulcus and superior medial frontal paracingulate, $t(23)=2.07, P<0.05$. No other comparison reached the significance level, except that the autism group showed higher correlation between the left dorsolateral prefrontal cortex and the right inferior temporal lobe, $t(7)=3.48, P<.05$.

\section{Discussion}

The results of the present study can be summarized as follows. First, the autism group showed less activation in the left hemisphere frontal regions, whereas the control group showed bilateral activation in the frontal regions. As is seen in Table 2, the autism group showed the same amount of activation as the control group in the right hemisphere but much less activation in the left hemisphere in the dorsolateral prefrontal cortex, the inferior frontal gyrus, and the posterior precentral sulcus. According to Smith and

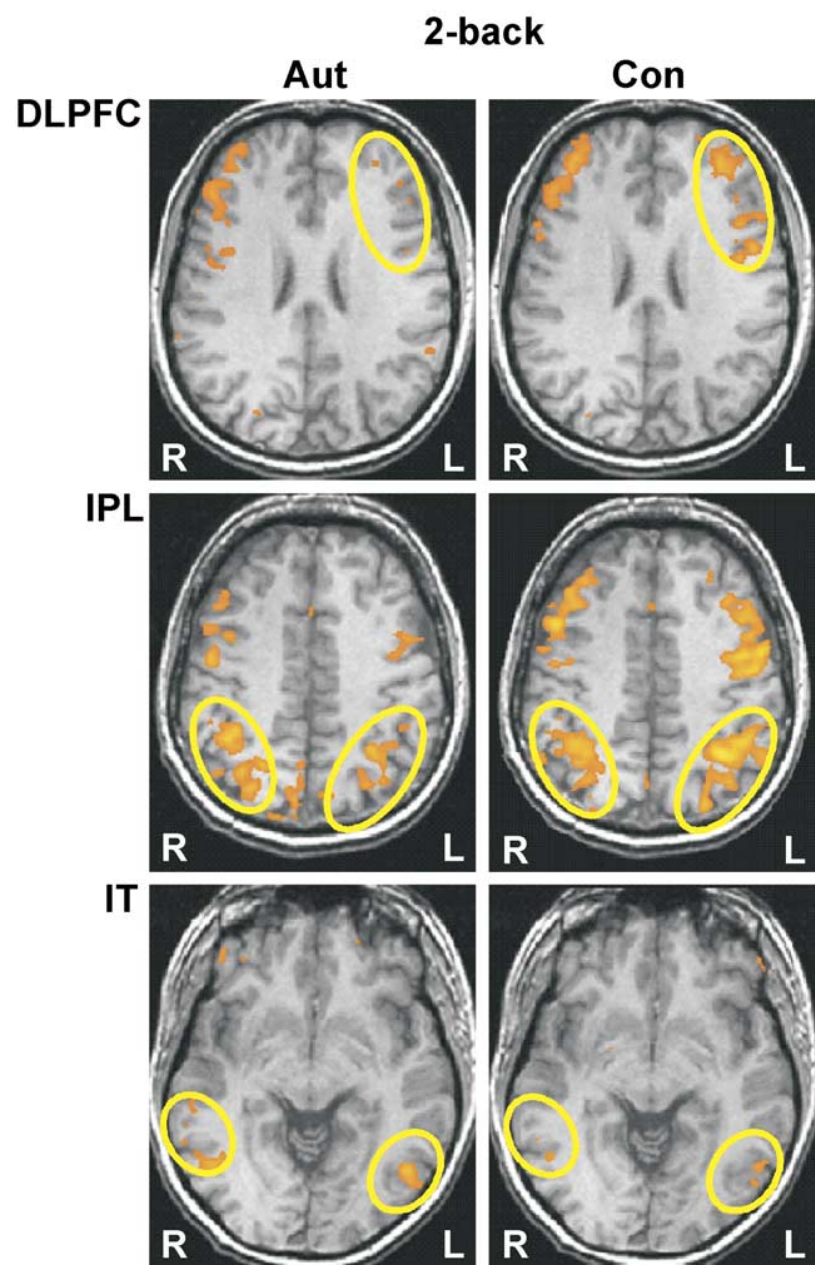

Fig. 4. $t$ maps that were transformed to a standardized space (Talairach and Tournoux, 1988) and averaged across participants using MCW-AFNI software (Cox, 1996) for the 2-back condition compared to the resting baseline. For the dorsolateral prefrontal cortex (DLPFC), the two groups showed the same level of activation in the right hemisphere, whereas the autism group showed much less activation than the control group in the left hemisphere. For the inferior parietal lobe (IPL), the autism group showed greater activation than the control group in the right hemisphere, whereas the autism group showed less activation than the control group in the left hemisphere. For the temporal lobe $(\mathrm{T})$, the autism group showed significantly greater activation than the control group. 


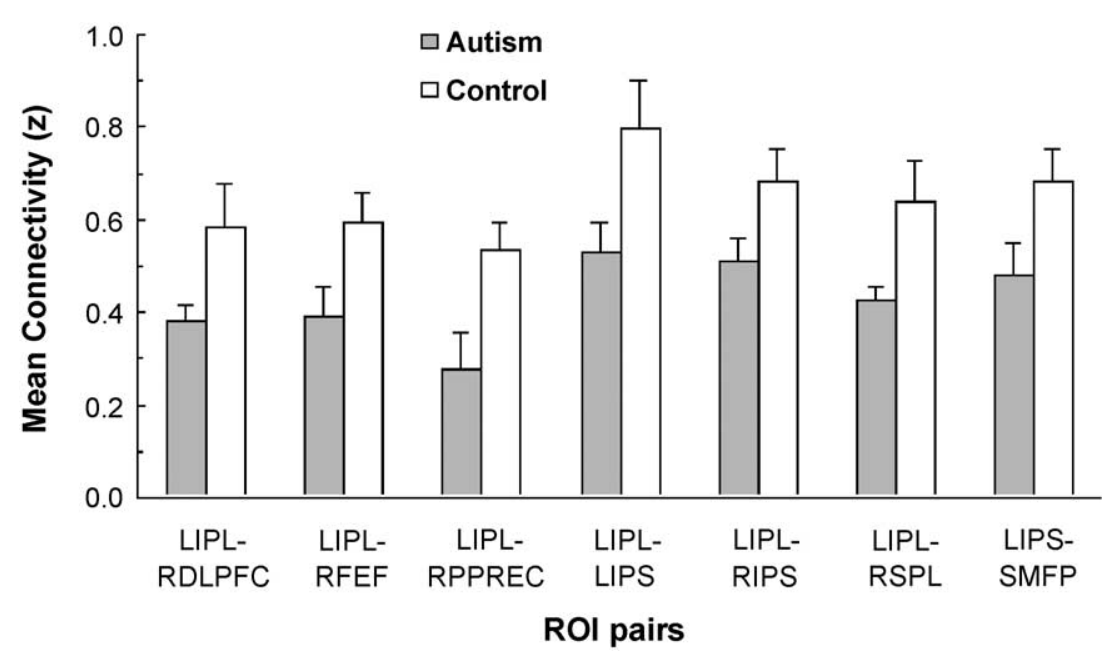

Fig. 6. The results of the group comparison including the ROI pairs that showed significant group differences in functional connectivity. One key finding is that the autism group showed lower connectivity between the left inferior parietal and the right prefrontal regions. LIPL indicates left inferior parietal lobe; LIPS, left intraparietal sulcus; RIFG, right inferior frontal gyrus; RFEF, right frontal eye field; RPPREC, right posterior precentral sulcus; RIPS, right intraparietal sulcus; RSPL, right superior parietal lobe; SMFP, bilateral superior medial paracingulate cortex.

parietal lobe which might be associated with the information buffer of working memory. In the inferior parietal lobe, the autism group showed more activation in the right hemisphere than left, whereas the control group showed more activation in the left than the right hemisphere. This difference possibly corresponds to the different information processing styles of the two groups. The control group might have used the expected verbal strategy in which they coded each stimulus letter verbally to facilitate memory. In other words, they showed more activation in the left inferior parietal regions because they used phonological codes to encode the stimulus letters. On the other hand, the autism group might have used a more nonverbal, visual-graphical approach (e.g., Posner, 1969) in which they coded the shapes of the alphabet letters without naming them, resulting in more activation in the right hemisphere. Together with the first point that the autism group showed hypoactivation in the left prefrontal cortex, these results seem to indicate that the autism group processed the letter stimuli as nonverbal, visualgraphical codes. The possibility of different information processing styles between the groups is also consistent with previous findings that individuals with autism tend to rely on nonverbal, visually

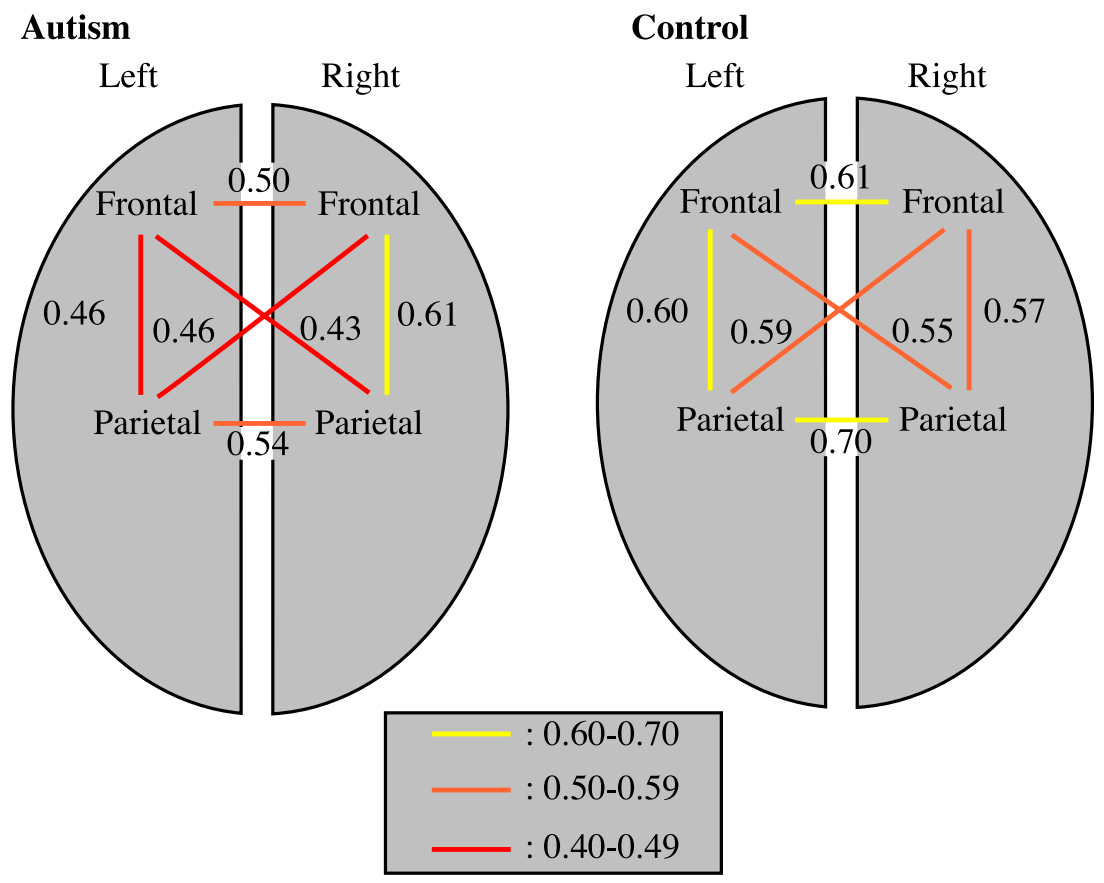

Fig. 7. The autism group tends to show the underconnectivity compared to the control group in the ROIs related to the working memory network, although the strength of connectivity also depends on the regions. For the autism group, the right parietal is more strongly correlated with the frontal regions than the left parietal is, whereas for the control group, the left parietal is more strongly correlated with the frontal regions than the right parietal. 
oriented information processing (e.g., Frith, 1989; Plaisted et al., 1998a,b, 1999; Ring et al., 1999; Shah and Frith, 1983).

Third, the autism group showed more activation in the posterior regions, including the left inferior temporal, left temporal, right temporal, and left inferior extrastriate, whereas the control group showed very little activation in these regions. This pattern might also be related to the information processing style of the participants with autism, suggesting that they relied on analysis of lower level visual features. In the present study, it is possible that the control group showed little activation in the posterior regions because the stimuli were letters which can be easily named; therefore, they used verbal coding, a highly automatic process for individuals with typical development.

These results from the analysis of the amount of activation are consistent with the results of the factor analyses. As is seen in Table 3, the factor analysis extracted three factors for the autism group. Factor 1 consists of the left and right prefrontal and right parietal ROIs, demonstrating greater synchronization between the prefrontal regions and right parietal regions. This result indicates that the working memory network for the autism group in the letter n-back task may consist of the prefrontal and the right parietal regions. Factor 3 contains the posterior (temporal and occipital) ROIs, and this corresponds to the greater amount of activation in the sum of signal intensity data in these regions. In contrast, the factor analysis extracted two factors for the control group. Factor 1 contains the left and right prefrontal and left parietal ROIs. This result indicates that the working memory network for the control group in the letter n-back task contains the prefrontal and left parietal regions, a finding that is consistent with the results of preceding working memory studies (e.g., Braver et al., 1997; Smith et al., 1998). Factor 2 includes the right inferior frontal gyrus and right parietal regions.

The results of the group comparison of the functional connectivity analysis are consistent with the results of the factor analyses. In the group comparison, the autism group showed lower correlations than the control group between the left inferior parietal and some frontal and parietal regions (see Fig. 6). These results again indicate that, unlike the control participants, the autism group might not encode the letter stimuli with phonological codes. These results are consistent with the underconnectivity theory of autism (Just et al., 2004) that proposes that individuals with autism tend to have lower functional connectivity than normal controls. However, the underconnectivity shown by the autism group in the present study was not general, but rather specific to particular regions. The underconnectivity was observed around the left parietal lobe, but other regions did not show much difference in correlation between the autism and control groups (see Fig. 7). This difference between the present study and Just et al.'s (2004) might result from a difference in the tasks. Just et al. (2004) used a sentence comprehension task, a task with which individuals with autism tend to have more difficulty. Therefore, it is possible that the underconnectivity depends on cortical regions and task requirements.

Taken together, the results from the analysis of the amount of activation, the factor analysis, and the group comparison of correlations all converge to suggest differences in information processing styles between the autism and control groups. Our interpretation of the pattern of results is that the autism group used a more nonverbal and visually oriented processing style and that they retained the stimuli as visual-graphical codes. The working memory network for the autism group therefore consists of the frontal and right parietal regions, as seen in their Factor 1. On the other hand, the control group relied on a verbally oriented style in which they converted the letter stimuli into verbal-phonological codes. Therefore, the working memory network for the control group includes the prefrontal and left parietal regions, as seen in their Factor 1.

The present data provide support for the three main hypotheses of this study. The first hypothesis was that individuals with autism would rely on lower level visuospatial feature analysis. This tendency was evidenced by the brain imaging results demonstrating that individuals with autism showed relatively less activation in the anterior regions and more activation in the posterior regions associated with visual processing. The second hypothesis that individuals with autism may process information in the right hemisphere more than the left hemisphere was also supported by the neuroimaging results. The third was that the large-scale brain network (e.g., Mesulam, 1990, 1998) of individuals with autism has a different organization from that of normal controls. The data from this study suggest that the working memory network for individuals with autism might be characterized as shifted toward the right hemisphere as well as toward the posterior part of the brain. Their weaker anterior (frontal) components may result from greater reliance on visual feature analysis, even in a verbal memory task such as the n-back letter task. The results of the present study are also consistent with the underconnectivity theory (Just et al., 2004), because the autism group showed lower synchronization among the brain areas than the control group in general. However, the present study also suggests that the degree of underconnectivity depends on task requirements and brain regions recruited for the task.

\section{Acknowledgments}

This research was supported by the National Institute of Child Health and Human Development (NICHD), grant number HD35469. The authors would like to thank Jacquelyn Cynkar, Jessica Hoge, Erika June Christina Laing, and Holly Zajac for assistance with the data collection and data analysis, and to Diane Williams for providing useful comments on the earlier version of the manuscript.

\section{References}

American Psychiatric Association Task Force on DSM-IV, 1994. Diagnostic and Statistical Manual of Mental Disorders, fourth ed. American Psychiatric Association, Washington, DC.

Bandettini, P.A., Wong, E., Hinks, R.S., Tokofsky, R.S., Hyde, J.S., 1992. Time course EPI of human brain function during task activation. Magn. Reson. Med. 25, 390-397.

Bennetto, L., Pennington, B.F., Rogers, S.J., 1996. Intact and impaired memory functions in autism. Child Dev. 67, 1816-1835.

Boddaert, N., Zilbovicius, M., 2002. Functional neuroimaging and childhood autism. Pediatr. Radiol. 32, 1-7.

Braver, T.S., Cohen, J.D., Nystrom, L.E., Jonides, J., Smith, E.E., Noll, D.C., 1997. A parametric study of prefrontal cortex involvement in human working memory. NeuroImage 5, 49-62.

Castelli, F., Frith, C., Happé, F., Frith, U., 2002. Autism, Asperger syndrome and brain mechanisms for the attribution of mental states to animated shapes. Brain 125, 1839-1849. 
Caviness Jr., V.S., Meyer, J., Makris, N., Kennedy, D.N., 1996. MRI-based topographic parcellation of human neocortex: an anatomically specified method with estimate of reliability. J. Cogn. Neurosci. 8, 566-587.

Cox, R.W., 1996. AFNI: software for visualization and analysis of functional magnetic resonance neuroimages. Comput. Biomed. Res. 29, 162-173.

Critchley, H.D., Daly, E.M., Bullmore, E.T., Williams, S.T.R., Amelsvoort, T.V., Robertson, D.M., Rowe, A., Phillips, M., McAlonan, G., Howlin, P., Murphy, D.G.M., 2000. The functional neuroanatomy of social behavior: changes in cerebral blood flow when people with Autism disorder process facial expressions. Brain 123, 2203-2212.

D'Esposito, M., 2001. Functional neuroimaging of working memory. In: Cabeza, R., Kingstone, A. (Eds.), Handbook of Functional Neuroimaging of Cognition. The MIT Press.

D’Esposito, M., Postle, B.R., Ballard, D., Lease, J., 1999. Maintenance versus manipulation of information held in working memory: an eventrelated fMRI study. Brain Cogn. 41, 66-86.

Eddy, W.F., Fitzgerald, M., Genovese, C.R., Mockus, A., Noll, D.C., 1996. Functional imaging analysis software-Computational olio. Proceedings in Computational Statistics. Physica-Verlag, Heidelberg, Germany, pp. $39-49$.

Fletcher, P.C., Henson, R.N.A., 2001. Frontal lobes and human memoryinsights from functional imaging. Brain 124, 849-881.

Friston, K.J., Frith, C.D., Liddle, P.F., Frackowiak, R.S.J., 1993. Functional connectivity: the principal-component analysis of large PET data sets. J. Cereb. Blood Flow Metab. 13, 5-14.

Frith, U., 1989. A new look at language and communication in autism. Br. J. Disord. Commun. 24, 123-150.

Hill, E.L., Frith, U., 2003. Understanding autism: insights from mind and brain. Philos. Trans. R. Soc. Lond., Ser. B 358, 281-289.

Hollingshead, A.B., 1957. Two-Factor Index of Social Position. Yale University Department of Sociology, New Haven, CT.

Just, M.A., Carpenter, P.A., Maguire, M., Diwadkar, V., McMains, S., 2001. Mental rotation of objects retrieved from memory: an fMRI study of spatial processing. J. Exp. Psychol. Gen. 130, 493-504.

Just, M.A., Cherkassky, V., Keller, T.A., Minshew, N.J., 2004. Cortical activation and synchronization during sentence comprehension in high-functioning autism: evidence of underconnectivity. Brain 127, $1811-1821$.

Kwong, K.K., Belliveau, J.W., Chesler, D.A., Goldberg, I.E., Weisskoff, R.M., Poncelet, B.P., Kennedy, D.N., Hoppel, B.E., Cohen, M.S., Turner, R., Cheng, H.-M., Brady, T.J., Rosen, B.R., 1992. Dynamic magnetic resonance imaging of human brain activity during primary sensory stimulation. Proc. Nat. Acad. Sci. U. S. A. 89, 5675-5679.

Lazar, N.A., Eddy, W.F., Genovese, C.R., Welling, J., 2001. Statistical issues in fMRI for brain imaging. Int. Stat. Rev. 69, 105-127.

Le Courteur, A., Rutter, M., Lord, C., Rios, P., Robertson, S., Holdgrafer, M., McLennan, J.D., 1989. Autism diagnostic interview: a semistructured interview for parents and caregivers of autistic persons. J. Autism Dev. Disord. 19, 363-387.

Lord, C., Rutter, M., Goode, S., Heemsbergen, J., Jordan, H., Mawhood, L., Schopler, E., 1989. Autism diagnostic observation schedule: a standardized observation of communicative and social behavior. J. Autism Dev. Disord. 19, 185-212.

Lord, C., Rutter, M., Le Couteur, A., 1994. Autism diagnostic interviewrevised: a revised version of a diagnostic interview for caregivers of individuals with possible pervasive developmental disorders. J. Autism Dev. Disord. 24, 659-685.

Lord, C., Cook, E.H., Leventhal, B.L., Amarel, D.G., 2000. Autism spectrum disorders. Neuron 28, 355-363.

Luna, B., Minshew, N.J., Garver, K.E., Lazar, N.A., Thulborn, K.R., Eddy, W.F., Sweeney, J.A., 2002. Neocortical system abnormalities in autism: an fMRI study of spatial working memory. Neurology 59, 834-840.

McIntosh, A.R., Gonzalez-Lima, F., 1994. Structural equation modeling and its application to network analysis in functional brain imaging. Hum. Brain Mapp. 2, 2-22.

McLaughlin, T., Steinberg, B., Christensen, B., Law, I., Parving, A., Friberg, L., 1992. Potential language and attentional networks revealed through factor analysis of $\mathrm{rCBF}$ data measured with SPECT. J. Cereb. Blood Flow Metab. 12, 535-545.

Mesulam, M.-M., 1990. Large-scale neurocognitive networks and distributed processing for attention, language and memory. Ann. Neurol. 28, 597-613.

Mesulam, M.-M., 1998. From sensation to cognition. Brain 121, $1013-1052$.

Minshew, N.J., 1996. Brief report: brain mechanisms in autism: functional and structural abnormalities. J. Autism Dev. Disord. 26, 205-209.

Minshew, N.J., Goldstein, G., Siegel, D.J., 1997. Neuropsychologic functioning in autism: profile of a complex information processing disorder. J. Int. Neuropsychol. Soc. 3, 303-316.

Mottron, L., Morasse, K., Belleville, S., 2001. A study of memory functioning in individuals with autism. J. Child Psychol. Psychiatry 42, $253-260$.

Muller, R.A., Behen, M.E., Rothermel, R.D., Chugani, D.C., Muzik, O., Mangner, T.J., Chugani, H.T., 1999. Brain mapping of language and auditory perception in high-functioning autistic adults: a PET study. J. Autism Dev. Disord. 29, 19-31.

Nieto-Castanon, A., Ghosh, S.S., Tourville, J.A., Guenther, F.H., 2003. Region of interest based analysis of functional imaging data. NeuroImage 19, 1303-1316.

Nystrom, L.E., Braver, T.S., Sabb, F.W., Delgado, M.R., Noll, D.C., Cohen, J.D., 2000. Working memory for letters, shapes, and locations: fMRI evidence against stimulus-based regional organization in human prefrontal cortex. NeuroImage 11, 424-446.

Ogawa, S., Lee, T.M., Kay, A.R., Tank, D.W., 1990. Brain magnetic resonance imaging with contrast dependent on blood oxygenation. Proc. Natl. Acad. Sci. U. S. A. 87, 9868-9872.

O'Riordan, M.A., Plaisted, K.C., Driver, J., Baron-Cohen, S., 2001. Superior visual search in autism. J. Exp. Psychol. Hum. Percept. Perform. 27, 719-730.

Owen, A.M., Stern, C.E., Look, R.B., Tracey, I., Rosen, B.R., Petrides, M., 1998. Functional organization of spatial and nonspatial working memory processing within the human lateral frontal cortex. Proc. Natl. Acad. Sci. U. S. A. 95, 7721-7726.

Ozonoff, S., Strayer, D.L., 2001. Further evidence of intact working memory in autism. J. Autism Dev. Disord. 31, 257-263.

Ozonoff, S., Strayer, D.L., McMahon, W.M., Filloux, F., 1994. Executive function abilities in autism and Tourette syndrome: an information processing approach. J. Child Psychol. Psychiatry 35, 1015-1032.

Peterson, B.S., Skudlarski, P., Gatenby, J.C., Zhang, H., Anderson, A.W., Gore, J.C., 1999. An fMRI study of Stroop word-color interference: evidence for cingulate subregions subserving multiple distributed attentional systems. Biol. Psychiatry 45, 1237-1258.

Petrides, M., 1994. Frontal lobes and working memory: evidence from investigations of the effects of cortical excisions in nonhuman primates. In: Boller, F., Grafman, J. Hand (Eds.), Handb. Neuropsychol., vol. 9. Elsevier, Amsterdam, pp. 59-82.

Pierce, K., Muller, R.A., Ambrse, J., Allen, G., Courchesne, E., 2001. Face processing occurs outside the fusiform 'Face Area' in Autism: evidence from functional MRI. Brain 124, 2059-2073.

Plaisted, K.C., O’Riordan, M., Baron-Cohen, S., 1998a. Enhanced discrimination of novel, highly similar stimuli by adults with autism during a perceptual learning task. J. Child Psychol. Psychiatry 39, 765-775.

Plaisted, K.C., O’Riordan, M., Baron-Cohen, S., 1998b. Enhanced visual search for a conjunctive target in autism: a research note. J. Child Psychol. Psychiatry 39, 777-783.

Plaisted, K.C., Swettenham, J., Rees, L., 1999. Children with autism show local precedence in a divided attention task and global precedence in a selective attention task. J. Child Psychol. Psychiatry 40, 733-742.

Posner, M.I., 1969. Abstraction and the process of recognition. In: Bower, G.H. (Ed.), Psychol. Learn. Motiv., vol. 3. Academic Press, New York.

Postle, B.R., 2000. "What" then "where" in visual working memory: an event-related fMRI study. J. Cogn. Neurosci. 11, 585-597.

Rademacher, J., Galaburda, A.M., Kennedy, D.N., Filipek, P.A., Caviness Jr., V.S., 1992. Human cerebral cortex: localization, parcellation, and 
morphometry with magnetic resonance imaging. J. Cogn. Neurosci. 4, $352-374$.

Reitan, R.M., 1985. Halstead-Reitan Neuropsychological Test Battery Reitan Neurological Laboratories, University of Arizona, Tucson, AZ.

Ring, H.A., Baron-Cohen, S., Wheelwright, S., Williams, S.C.R., Brammer, M., Andrew, C., Bullmore, E.T., 1999. Cerebral correlates of preserved cognitive skills in autism. Brain 122, 1305-1315.

Russell, J., Jarrold, C., Henry, L., 1996. Working memory in children with autism and with moderate learning difficulties. J. Child Psychol. Psychiatry 37, 673-686.

Schultz, R.T., Gauthier, I., Klin, A., Fulbright, R.K., Anderson, A.W., Volkmar, F., Skudlarski, P., Lacadie, C., Cohen, D.J., Gore, J.C., 2000 Abnormal ventral temporal cortical activity during face discrimination among individuals with autism and Asperger syndrome. Arch. Gen. Psychiatry $57,331-340$.

Shah, A., Frith, U., 1983. An islet of ability in autistic children: a research note. J. Child Psychol. Psychiatry 24, 613-620.

Shah, A., Frith, U., 1993. Why do autistic individuals show superior performance on the block design task? J. Child Psychol. Psychiatry 34, $1351-1364$.

Siegel, D.J., Minshew, N.J., Goldstein, G., 1996. Wechsler IQ profiles in diagnosis of high-functioning autism. J. Autism Dev. Disord. 26, $389-406$

Smith, E.E., Jonides, J., 1999. Storage and executive processes in the frontal lobes. Science 283, 1657-1661.

Smith, E.E., Jonides, J., Marshuetz, C., Koeppe, R., 1998. Components of verbal working memory: evidence from neuroimaging. Proc. Natl. Acad. Sci. U. S. A. 95, 876-882.

Talairach, J., Tournoux, P., 1988. Co-Planar Stereotaxic Atlas of the Human Brain. Thieme Medical Publishers, New York.

Thulborn, K.R., Voyvodic, J., McCurtain, B., Gillen, J., Chang, S., Just, M.A., Carpenter, P.A., Sweeney, J.A., 1996. High field functional MRI in humans: applications to cognitive function. In: Pavolone, P.P., Rossi, P. (Eds.), Proceedings of the European Seminars in Diagnostic and Interventional Imaging. Springer-Verlag, New York, pp. 91-96.

Ungerleider, L.G., Haxby, J., 1984. 'What' and 'where' in the human brain. Curr. Opin. Neurobiol. 4, 157-165.

Williams, D.L., Goldstein, G., Carpenter, P.A., Minshew, N.J., in pressa. Verbal and spatial working memory in autism. J. Autism Dev. Disord.

Williams, D.L., Goldstein, G., Minshew, N.J., in pressb. Impaired memory for faces and social scenes in autism: clinical implications of memory dysfunction. Arch. Clin. Neuropsychol. 